\title{
New animal-based measures to assess welfare in dromedary camels
}

\author{
Laura Menchetti $^{1} \cdot$ Bernard Faye $^{2,3} \cdot$ Barbara Padalino $^{1}[$
}

Received: 24 May 2021 / Accepted: 21 October 2021 / Published online: 5 November 2021

(c) The Author(s), under exclusive licence to Springer Nature B.V. 2021

\begin{abstract}
Reliable and measurable animal-based measures (ABMs) are essential for assessing animal welfare. This study aimed at proposing ABMs for dromedary camels identifying their possible associations with management. Data were collected at a permanent camel market; a total of 76 pens and 528 camels were evaluated. ABMs were collected for each welfare principle (i.e., good feeding, good housing, good health, appropriate behavior), while resources or management-based measures were collected at three levels of investigations (animal, herd, or caretakers). Associations were calculated by generalized linear models. Body condition score and thirst index (ABMs of good feeding) resulted negatively associated with short caretaker's experience, dirty bedding, limited shaded space, feeding and water space, and space allowance $(P<0.05)$. Resting behaviors and restricted movements (ABMs of good housing) were associated with short caretaker's experience, dirty bedding and water, rationed water distribution, water points in the sun, and presence of hobbles $(P<0.05)$. Disease, injury, and pain induced by management procedures (ABMs of good health) were negatively associated with short caretaker's experience, presence of hobbles, limited space allowance and shaded space, dirty bedding, and feeding and watering practices (e.g., frequency of distribution, resource quality, location of the troughs; $P<0.05$ ). Response to approaching test and aggressivity (ABMs of Appropriate behavior) were negatively associated with limited space allowance, shaded, feeding and water space, and rationed water distribution $(P<0.05)$. Overall, the proposed ABMs seems to be appropriate indicators of welfare consequences in camels being able to identify factors related to housing and management practices that may impair or improve camel welfare.
\end{abstract}

Keywords Feeding $\cdot$ Housing $\cdot$ Health $\cdot$ Behavior $\cdot$ Management factor

\section{Introduction}

Animal welfare measures how an animal is coping with the environment where it lives (Broom, 1996; OIE, 2008). Animal welfare assessment consequently has to be based on reliable, measurable, and valid indicators. Those measures may be animal-based (ABMs) or resource and management-based indicators. Often, ABMs reflect the consequences, positive or negative, of factors related to

Barbara Padalino

barbara.padalino@unibo.it

1 Dipartimento Di Scienze E Tecnologie Agro-Alimentari, Alma Mater Studiorum, Università Di Bologna, Bologna, Italy

2 UMR SELMET, CIRAD-ES, Baillarguet International Campus, 34098 Montpellier Cedex, France

3 Al-Farabi Kazakh National University, 050040 Almaty, Kazakhstan housing and management and are therefore treated as the outcome in the formal risk assessment analysis (EFSA, 2012c; a). In the last decade, scientists have emphasized the role of ABMs since they can better express how an animal is feeling both physically and psychologically providing an integrative and direct measurement of its welfare state (Broom, 1996; Main et al., 2007; Munoz et al., 2018). ABMs may indeed indicate not only impaired functioning associated with malnutrition, dehydration, movement difficulties, injury, and disease but also needs and affective states such as pain and fear (Broom, 1996; EFSA, 2012a). The ABMs selected to obtain an impression of the animal's quality of life as complete as possible should cover all dimensions of animal welfare, reflecting thus the multidimensional concept of welfare. According to the Welfare Quality ${ }^{\circledR}$ and AWIN projects, four main dimensions of welfare, called principles, should be monitored: good feeding, good housing, good health, and appropriate behavior (Welfare Quality, 2009; AWIN, 2015). The 
ABMs, however, are required to be not only reliable but also feasible in order to make the on-farm assessment protocol both time- and resource-saving (Main et al., 2007; EFSA, 2012c). A short-list of ABMs could be selected avoiding overlapping of information but covering the main factors capable of inducing concerns for the welfare of that particular animal (EFSA, 2012c). In this context, an important feature of ABMs is their "fitness for the purpose of the assessment." The selected ABMs should be fit for that particular animal species and category at that time, for the skills of assessors, the conditions under which they are to be collected, ethics, and financial constraints (Main et al., 2007; EFSA, 2012c).

The attention of the scientific world has therefore focused on the selection of ABMs according to the species and the farming systems. However, most of the research has been conducted in European countries for animals such as pigs, poultry, ewe, and dairy cattle (Main et al., 2007; EFSA, 2012b; Cook, 2017; Munoz et al., 2018; Tremolada et al., 2020). Among farm animals, the camel was rarely involved in studies related to welfare assessment (Pastrana et al., 2020; Zappaterra et al., 2021) and an ad hoc protocol for its welfare assessment has only recently been proposed (Padalino and Menchetti, 2021). This protocol includes a combination of animal-, resource-, and management-based measures assessed at three levels of investigation: using a face-to-face interview with the caretaker (caretaker level), checking the herd and the pen facilities (herd level), and inspecting individual camel behavior and health status (animal level). These measures could be then scored and combined to produce overall assessment indices, and to classify the pens according to their camel welfare level (Menchetti et al., 2021). As far as we know, however, no specific ABMs have been selected for camels and associated with a variety of possible factors collected at caretaker, herd, and animal levels of investigation. Their relationship with factors that may affect camel welfare, moreover, has not been investigated yet in a thorough manner embracing the principles of good feeding, good housing, good health, and appropriate behavior. These topics should be urgently addressed as important steps towards the identification of welfare concerns and standards for camels. With the intensification of camel production in many parts of the world (Bengoumi and Faye, 2002; Faye, 2014), indeed, the possible harms to the well-being of these animals in intensive farms or transport are increasing (Bauer and Havenstein, 2017; Pastrana et al., 2020).

Thus, hypothesizing that ABMs would be related to management, the aim of this study was to propose new ABMs for dromedary camels and to evaluate the possible associations between these ABMs, used as indicators of welfare consequences, and resource and management-related factors in camels kept in an intensive system.

\section{Materials and methods}

\section{General description of housing and animals}

This study was conducted as part of a larger study aimed at applying a protocol for the welfare assessment of camels kept at the permanent camel market in Qatar. The research project was run with the permission of the Department for Agriculture Affairs and Fisheries of the Ministry of Municipality and Environment of the State of Qatar (protocol code 2404/2020). The study involved no invasive sampling methods and all data collection was performed without disturbing the animals. Oral owners' and caretakers' consents were received before the assessment.

Data were collected at the market in the morning (7:00-11:00 AM) from the 11th to the 18th of September 2019. The mean temperature and humidity were $42.3{ }^{\circ} \mathrm{C}$ (standard deviation $\left.(\mathrm{SD})=2.9^{\circ} \mathrm{C}\right)$ and $32.2 \%(\mathrm{SD}=8.6 \%)$, respectively, while temperature humidity index (THI) was $89(\mathrm{SD}=3)$. The Doha market is a permanent market where there are 92 pens; 76 out of 92 pens contained animals at the time of the study and were therefore involved in our data collection, with a total of 528 camels. Camels could have different owners and caretakers; they came from different geographical areas (i.e., Qatar, Sudan, Oman, Saudi Arabia, United Arab Emirates, Kuwait, Pakistan, and Somalia) and were kept at the market for different purposes (i.e., milk, meat, breeding, sales, or racing).

\section{Measures, data collection, and handling}

The assessment was conducted by five assessors with a solid scientific background in camel behavior, health, and welfare using three different approaches (i.e., three levels of investigation, caretaker, herd, and animal level). This approach, the entire protocol, and procedures for data collection were developed in 2019 but published and described in detail later by Padalino and Menchetti (2021). The caretaker level consisted of a face-to-face interview with 49 caretakers who managed one or more pens at the market. Four of the 23 items of the questionnaire proposed by the protocol of Padalino and Menchetti (2021) were used in the present study. The herd level consisted of a check of the general characteristics of the pens ( $n=76$ pens) and all animals included therein ( $n=528$ camels). Seventeen measures among those proposed by the protocol (Padalino and Menchetti, 2021) were selected for the present study. The animal level consisted of a deeper inspection of 2 randomly selected animals within each pen, but in some pens, there was only one camel ( $n=132$ camels). The authors could perform the deeper inspection of a maximum of two camels per pen, due 
to constraints in animal ethics. Eleven measures among those proposed for the Animal level by the protocol (Padalino and Menchetti, 2021) were selected for the present study. Each level of investigation included both animal-based and management- or resource-based measures.

The animal-based measures treated as welfare consequences were all "direct" indicators (i.e., taken from the animal (EFSA, 2012c)) and they did not require further laboratory analysis. At least two indicators for each welfare principle (i.e., good feeding, good housing, good health, and appropriate behavior) were selected, as detailed in Table 1 . Animal-based measures could be collected at both herd and animal levels, according to the protocol of Padalino and Menchetti (2021). Some measures involved only the visual inspection of the animals (e.g., body condition score (BCS), presence of disease or injuries) while others required behavioral observations (i.e., resting behavior and aggressivity) or specific behavioral tests (i.e., Thirst Index and Approaching test). For the presence of disease, evident clinical signs, such as swollen joints or udder, lameness, discharge (i.e., nasal, ocular, vulvar), diarrhea, alopecia, coughing, and abnormal breathing, were noted down. For statistical purposes and due to the skewed data distribution, measures expressed as scores (i.e., BCS and thirst index) and a measure collected at Herd level (i.e., aggressivity) were categorized in 2 or 3 categories (Table 1). The other measures collected at the herd level (i.e., restricted movements and pain induced by management procedures) were, instead, expressed as the proportion of animals in the pen. As the number of animals in the pen showed great variability (from 1 to 37 camels) and the proportion could be biased in uncrowded pens (e.g., $100 \%$ indicated only one affected animal in pens holding 1 camel), each percentage was weighted for the maximum number of animals for which the criterion was satisfied.

The presence of stereotypies, initially selected as welfare consequence for appropriate behavior, was instead excluded from the analysis as no events were recorded.

Management- and resource-based measures were treated as factors, because they could have the potential to influence welfare, and were instead collected at all three levels of investigation (Table 2). At the caretaker level, information mainly related to management, such as the feeding, watering, and health management practices, and the camels' productive purpose were obtained. The camels' productive purpose could indeed have influenced the approach of the caretaker and his management choices. Furthermore, assuming that the experience of the caretakers could influence management choices and their ability in approaching/handling animals (des Roches et al., 2016; Diverio et al., 2016; Menchetti et al., 2020), a question related to the caretaker's experience was included as a possible factor. Caretakers' experience was later categorized into three categories (Table 2). Measures collected at the herd level were mainly related to space allocation and housing facilities (i.e., space allowance, shade availability, number of water and feeding points, water and feeding space per animal, water and feed quality, cleanliness of bedding). Other management-based measures involved the observation of the camels within the pen and they could be collected both at herd and animal levels (i.e., the presence of tethered and hobbled animals). The measures could be expressed as categorical (e.g., clean, partially clean or dirty; sun or shade; presence/absence of hobbles) or numerical (e.g., the number of feeding and water points, water temperature) variables. However, for statistical purposes, some numerical variables (e.g., space allowance, water, and feeding space) were categorized by statistical binning using the tertiles as the threshold (Altman, 2014; Menchetti et al., 2021).

Moreover, some animal-based measures (i.e., age, presence of diseases or injuries) were also treated as factors since they could have influenced some welfare outcomes (Table 2).

\section{Statistical analysis}

Descriptive statistics were used to present the collected measures using mean and standard deviation (SD), median (Mdn), and range or interquartile range (IQR), number, and percentage. The observed distributions of categorical variables (except those obtained by statistical binning) were compared with the expected probability distributions (each assuming all categories equal) using the chi-square goodness of fit test.

Associations between $\mathrm{ABMs}$ and management or resource-related factors were evaluated by the specific statistical models reported in Table 1. Each ABM (Table 1) was included as a dependent variable while measures reported in Table 2 were included as predictors. When the dependent variable was evaluated at the animal level and there was no multicollinearity, "age of camel" was included in the models as a covariate. Multicollinearity was verified by Chi-square or Kruskal-Wallis tests and it was found for the following variables: number of camels in the pen, space allowance, feeding, and water space per animal. "Camel" was included in the models as "subject" while "pen" was included as within-subject variable (as two camels per pen were evaluated). Codes used for each category are also detailed in Table 1 . The first category (i.e., 0 ) was set as the reference category. Results were expressed as odds ratio (OR), 95\% confidence interval $(95 \% \mathrm{CI})$, and the $P$-value of Wald statistic. The estimated marginal means and standard errors were also reported for the results of interest.

Statistical analyses were performed using SPSS 25.0 (SPSS, an IBM Company, Chicago, IL) while GraphPad Prism, version 7.0 (GraphPad Software, San Diego, CA), was used for visualization. Statistical significance occurred when $P \leq 0.05$ but the trends towards significance $(P<0.1)$ were also reported and discussed. 


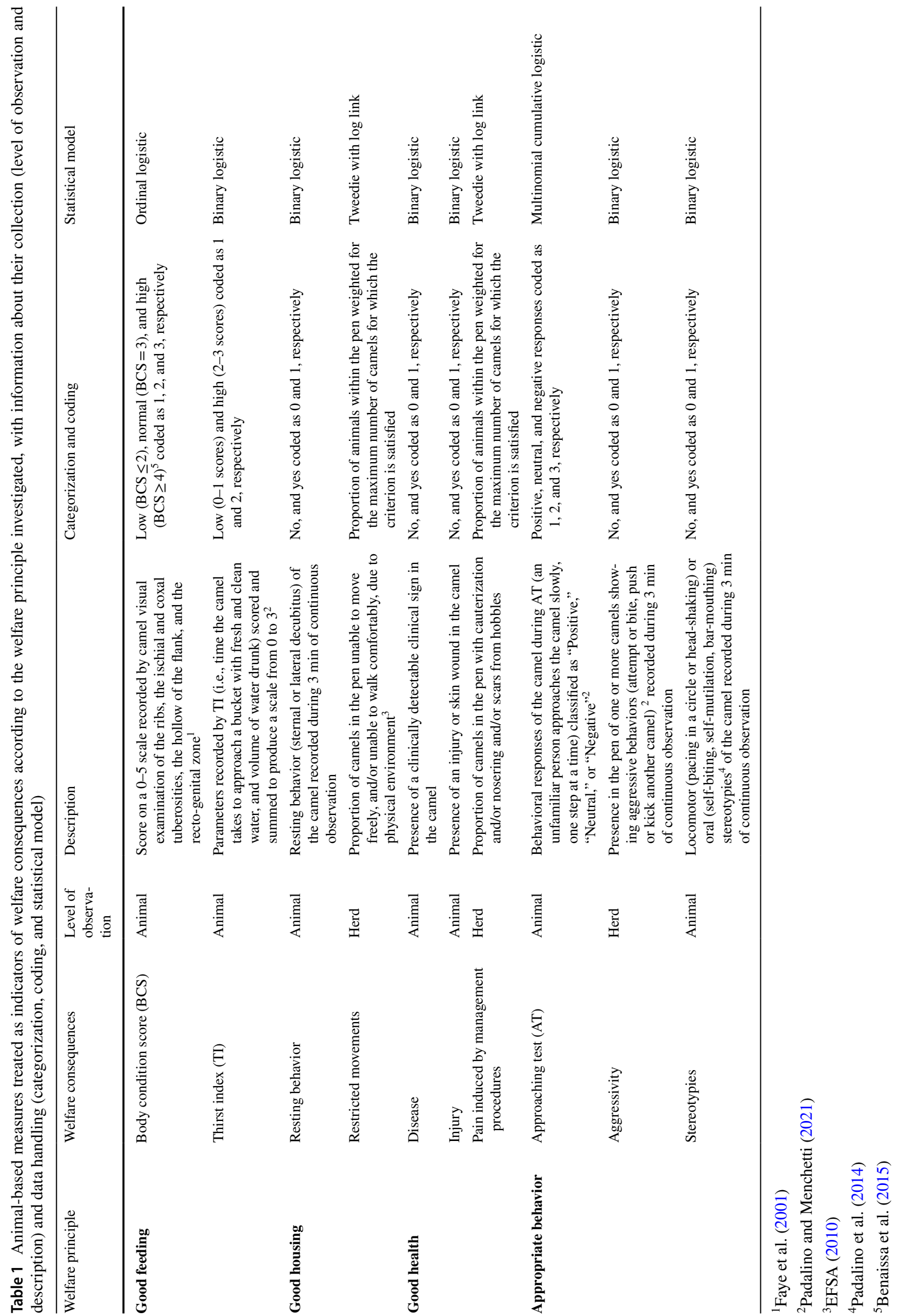


Table 2 Measures included in the protocol of Padalino and Menchetti (2021) and in the present study treated as possible factors with the ability to impair or improve camel welfare. They were included in the models as predictors with their level of investigation and type of variable or their categories

\begin{tabular}{|c|c|c|}
\hline Measure & Level of investigation & Type of variable/categories \\
\hline Frequency of feed distribution & Caretaker & $\begin{array}{l}\text { Ad libitum } \\
\text { Rationed }\end{array}$ \\
\hline Frequency of water distribution & Caretaker & $\begin{array}{l}\text { Ad libitum } \\
\text { Rationed }\end{array}$ \\
\hline Productive purposes of camels & Caretaker & $\begin{array}{l}\text { Meat } \\
\text { Milk, breeding, or race } \\
\text { Both }\end{array}$ \\
\hline $\begin{array}{l}\text { Caretaker's experience in working with } \\
\text { camels }\end{array}$ & Caretaker & $\begin{array}{l}>10 \text { years (well-experienced) } \\
6-10 \text { years (moderately experienced) } \\
0-5 \text { years (slightly experienced) }\end{array}$ \\
\hline Space allowance & Herd & $\begin{array}{l}\leq 19.0 \mathrm{~m}^{2} / \text { camel (limited) } \\
19.1-40.0 \mathrm{~m}^{2} / \text { camel (regular) } \\
>40.0 \mathrm{~m}^{2} / \text { camel (ample) }\end{array}$ \\
\hline Shaded space allowance & Herd & $\begin{array}{l}\leq 2.50^{2} \mathrm{~m}^{2} / \text { camel (limited) } \\
2.51-7.00 \mathrm{~m}^{2} / \text { camel (regular) } \\
>7.00 \mathrm{~m}^{2} / \text { camel (ample) }\end{array}$ \\
\hline Water space per animal & Herd & $\begin{array}{l}\leq 0.060^{2} \mathrm{~m}^{2} / \text { camel (limited) } \\
0.061-0.160 \mathrm{~m}^{2} / \text { camel (regular) } \\
>0.160 \mathrm{~m}^{2} / \text { camel (ample) }\end{array}$ \\
\hline Water quality ${ }^{1}$ & Herd & $\begin{array}{l}\text { Dirty (D) } \\
\text { Partially clean or clean (PC/C) }\end{array}$ \\
\hline Water point location ${ }^{1}$ & Herd & $\begin{array}{l}\text { Sun } \\
\text { Shade }\end{array}$ \\
\hline Water temperature & Herd & Continuous variable \\
\hline Feeding space per animal & Herd & $\begin{array}{l}\leq 0.40^{¥} \mathrm{~m}^{2} / \text { camel (limited) } \\
0.41-1.10 \mathrm{~m}^{2} / \text { camel (regular) } \\
>1.10 \mathrm{~m}^{2} / \text { camel (ample) }\end{array}$ \\
\hline Feed quality ${ }^{1}$ & Herd & $\begin{array}{l}\text { Dirty (D) } \\
\text { Partially clean or clean (PC/C) }\end{array}$ \\
\hline Feeding point location ${ }^{1}$ & Herd & $\begin{array}{l}\text { Sun } \\
\text { Shade }\end{array}$ \\
\hline Cleanliness of bedding & Herd & $\begin{array}{l}\text { Dirty (D) } \\
\text { Partially clean or clean (PC/C) }\end{array}$ \\
\hline Number of camels in the pen & Herd & Continuous variable \\
\hline Tethering and hobbles & Animal or herd & No/yes or continuous variable \\
\hline $\mathrm{Age}^{3}$ & Animal & Continuous variable \\
\hline Disease $e^{3,4}$ & Animal or herd & No/yes or continuous variable \\
\hline Injury ${ }^{3,4}$ & Animal or herd & No/yes or continuous variable \\
\hline
\end{tabular}

${ }^{1}$ Referred to a randomly selected trough (when more than one trough was present).

${ }^{2}$ Or not available (i.e., $0 \mathrm{~m}^{2} /$ camel).

${ }^{3}$ Animal-based measures.

${ }^{4}$ Treated both as dependent and predictor variable.

\section{Results}

\section{Descriptive statistics of measures and prevailing categories}

The descriptive statistics of the measures collected at caretaker level are shown in Table 3. Rationed feeding was mostly applied $(P<0.001)$ while water was rationed in about half of the pens $(P=0.491)$. The majority of the animals were kept for milk production, breeding, or race $(P<0.001)$ and about half of the pens were managed by well-experienced caretakers $(P=0.029)$.

According to the descriptive statistics of the measures collected at herd level (Tables 4 and 5), the median number of camels per pen was 5 , but $25 \%$ of the pens included more than 8 camels. The space allowance ranged from 3 to $161 \mathrm{~m}^{2} /$ animal $\left(\mathrm{Mdn}=24 \mathrm{~m}^{2} /\right.$ animal $)$. Shelters were present in most of the pens $(85.5 \%, P<0.001)$ but the shaded 
Table 3 Descriptive statistics and relative goodness of fit test of the measures collected at caretaker level. Values are absolute $(\mathrm{N})$ and relative $(\mathrm{N} \%)$ frequency $(n=76$ pens)

\begin{tabular}{lllll}
\hline Measure & Category & $\mathrm{N}$ & $\mathrm{N} \%$ & $P$ value $^{1}$ \\
\hline Frequency of feed distribution & Ad libitum & 15 & $19.7 \%$ & $<0.001$ \\
& Rationed & $61^{*}$ & $80.3 \%$ & \\
Frequency of water distribution & Ad libitum & 35 & $46.1 \%$ & 0.491 \\
& Rationed & 41 & $53.9 \%$ & \\
Productive purposes of camels & Meat & 11 & $14.7 \%$ & $<0.001$ \\
& Milk, breeding or race & $40^{*}$ & $53.3 \%$ & \\
\multirow{2}{*}{ Caretaker's experience in working } & Both & 24 & $32.0 \%$ & \\
with camels & Well-experienced & $36^{*}$ & $47.4 \%$ & 0.029 \\
& Moderately experienced & 22 & $28.9 \%$ & \\
\hline
\end{tabular}

${ }^{1} P$ value from chi-square goodness of fit test.

*Higher observed number respect to expected (all categories equal). space allowance was less than $2.50 \mathrm{~m}^{2} / \mathrm{animal}$ in one-third of the pens. Median water and feeding space per animal were instead 0.1 and $0.65 \mathrm{~m}^{2} /$ animal, respectively, but there were 7 pens without water points and 1 pen without feeding point. Moreover, both water $(P<0.001)$ and feeding $(P<0.001)$ points were mainly positioned in the sun and the water temperature was higher than $38.3{ }^{\circ} \mathrm{C}$ in about $25 \%$ of the pens. Finally, in about a third of pens, water $(P=0.001)$ and feed $(P=0.066)$ were classified as dirty. In most of the pens, bedding was clean or partially clean $(P=0.004)$. In half of the pens, the prevalence of disease ranged from 12.5 to $66.7 \%$. A lower prevalence was found for injuries but a high percentage of camels showed signs of pain induced by management procedures. The proportion of camels with restricted movements and showing aggressive behaviors was very variable, ranging from 0 to $100 \%$.

At animal level (Table 6), more than $60 \%$ of the camels had a BCS $<3(P<0.001)$ and showed the worst scores for the thirst index $(P=0.001)$. Only 5 camels were tethered but about $20 \%$ were hobbled $(P<0.001)$. At animal level, the prevalence of diseases and injuries was $38.6 \%$ $(P=0.009)$ and $22.0 \%(P<0.001)$, respectively. About half of the animals examined showed resting behavior $(P=0.296)$ and a positive response to the approaching test $(P<0.001)$. Furthermore, stereotypical behavior was never noted $(P<0.001)$.

\section{Associations between animal-based measures, as indicators of welfare consequences, and management or resource-based measures, as factors that may affect welfare}

\section{Good feeding (Fig. 1a and b)}

Camel's age, as a result of both univariable and multivariable models (for all OR $>1.00 ; P<0.001$ ), was one of the
Table 4 Descriptive statistics and relative goodness of fit test of the measures collected at herd level and evaluated as categorical variables. All measures were management- or resourced-based. Values are absolute $(\mathrm{N})$ and relative $(\mathrm{N} \%)$ frequency ( $n=76$ pens)

\begin{tabular}{|c|c|c|c|c|}
\hline Measure & Category & $\mathrm{N}$ & $\mathrm{N} \%$ & $P$ value $^{1}$ \\
\hline \multirow[t]{3}{*}{ Space allowance ${ }^{3}$} & Limited & 26 & $34.2 \%$ & \multirow[t]{3}{*}{-} \\
\hline & Regular & 25 & $32.9 \%$ & \\
\hline & Ample & 25 & $32.9 \%$ & \\
\hline \multirow{3}{*}{$\begin{array}{l}\text { Shaded space } \\
\text { allowance }^{3}\end{array}$} & Limited & 23 & $30.3 \%$ & \multirow[t]{3}{*}{-} \\
\hline & Regular & 27 & $35.5 \%$ & \\
\hline & Ample & 26 & $34.2 \%$ & \\
\hline \multirow{3}{*}{$\begin{array}{l}\text { Water space per } \\
\text { animal }^{3}\end{array}$} & Limited & 22 & $28.9 \%$ & \multirow[t]{3}{*}{ - } \\
\hline & Regular & 27 & $35.5 \%$ & \\
\hline & Ample & 27 & $35.5 \%$ & \\
\hline \multirow[t]{2}{*}{ Water quality ${ }^{2}$} & Dirty & 25 & $32.9 \%$ & \multirow[t]{2}{*}{0.001} \\
\hline & $\begin{array}{l}\text { Partially clean or } \\
\text { Clean }\end{array}$ & $51^{*}$ & $67.1 \%$ & \\
\hline \multirow[t]{2}{*}{ Water point location ${ }^{2}$} & Sun & $66^{*}$ & $95.7 \%$ & \multirow[t]{2}{*}{0.029} \\
\hline & Shade & 3 & $4.3 \%$ & \\
\hline \multirow{3}{*}{$\begin{array}{l}\text { Feeding space per } \\
\text { animal }^{3}\end{array}$} & Limited & 26 & $34.2 \%$ & \multirow[t]{3}{*}{-} \\
\hline & Regular & 24 & $31.6 \%$ & \\
\hline & Ample & 26 & $34.2 \%$ & \\
\hline \multirow[t]{2}{*}{ Feed quality ${ }^{2}$} & Dirty & 30 & $39.5 \%$ & \multirow[t]{2}{*}{0.066} \\
\hline & $\begin{array}{l}\text { Partially clean or } \\
\text { Clean }\end{array}$ & 46 & $60.5 \%$ & \\
\hline \multirow{2}{*}{$\begin{array}{l}\text { Feeding point } \\
\text { location }^{2}\end{array}$} & Sun & $62 *$ & $82.7 \%$ & \multirow[t]{2}{*}{0.029} \\
\hline & Shade & 13 & $17.3 \%$ & \\
\hline \multirow{2}{*}{$\begin{array}{l}\text { Cleanliness of bed- } \\
\text { ding }\end{array}$} & Dirty & 12 & $15.8 \%$ & \multirow[t]{2}{*}{0.004} \\
\hline & $\begin{array}{l}\text { Partially clean or } \\
\text { Clean }\end{array}$ & $64 *$ & $84.2 \%$ & \\
\hline
\end{tabular}

${ }^{1} P$ value from chi-square goodness of fit test. Not calculated for categories obtained with statistical binning (-).

${ }^{2}$ Referred to a randomly selected trough (when more than one trough was present).

${ }^{3}$ Chi-square goodness of fit test not performed as the measure had been categorized using statistical binning (equal percentile).

*Higher observed number respect to expected (all categories equal). 
Table 5 Descriptive statistics of the measures collected at herd level and evaluated as numerical variables. The list includes both animal-based and managementor resourced-based measures. Values are median (Mdn), interquartile range (IQR), and range (minimum and maximum)

\begin{tabular}{llcll}
\hline Measure & Median & IQR & Minimum & Maximum \\
\hline Animals/pen (number) & 5 & $3-8$ & 1 & 37 \\
Proportion of camels with a disease (\%) & 40.00 & $12.5-66.7$ & 0.0 & 100.0 \\
Proportion of camels with physical injuries $(\%)$ & 0.0 & $0.0-7.3$ & 0.0 & 100.0 \\
Proportion of camels with restricted movements ${ }^{1}(\%)$ & 0.0 & $0.0-20.0$ & 0.0 & 100.0 \\
Proportion of camels with pain induced by management & 58.3 & $25.0-100.0$ & 0.0 & 100.0 \\
$\quad$ procedures ${ }^{2}(\%)$ & & & & \\
Proportion of camels showing aggressive behaviors $(\%)$ & 0.0 & $0.0-80.0$ & 0.0 & 100.0 \\
Water temperature $\left({ }^{\circ} \mathrm{C}\right)$ & 35.6 & $32.6-38.3$ & 32.0 & 43.0 \\
\hline
\end{tabular}

${ }^{1}$ Camels unable to move freely, and/or unable to walk comfortably, due to physical environment.

${ }^{2}$ Cauterization and/or nosering and /or scars from hobbles.
Table 6 Descriptive statistics and relative goodness of fit test of the categorical measures collected at animal level. Values are absolute $(\mathrm{N})$ and relative $(\mathrm{N} \%)$ frequency $(n=132 \mathrm{camel})$

\begin{tabular}{lllll}
\hline Measure & Category & $\mathrm{N}$ & $\mathrm{N} \%$ & $P$ value $^{1}$ \\
\hline Body Condition Score & Low & $83^{*}$ & $62.9 \%$ & $<0.001$ \\
& Normal & 30 & $22.7 \%$ & \\
& High & 19 & $14.4 \%$ & \\
Thirst Index & Low & 47 & $35.9 \%$ & 0.001 \\
& High & $84^{*}$ & $64.1 \%$ & \\
Tethering (presence) & No & $127^{*}$ & $96.2 \%$ & $<0.001$ \\
& Yes & 5 & $3.8 \%$ & \\
Hobbles (presence) & No & $105^{*}$ & $79.5 \%$ & $<0.001$ \\
& Yes & 27 & $20.5 \%$ & \\
Disease (presence) & No & $81^{*}$ & $61.4 \%$ & 0.009 \\
Injury (presence) & Yes & 51 & $38.6 \%$ & \\
& No & $103^{*}$ & $78.0 \%$ & $<0.001$ \\
Stereotypies (presence) & Yes & 29 & $22.0 \%$ & \\
Resting behavior (presence) & No & 132 & $100.0 \%$ & - \\
& Yes & 60 & $54.5 \%$ & 0.296 \\
Approaching test (response) & Positive & $62 *$ & $48.1 \%$ & 0.001 \\
& Neutral & 38 & $29.5 \%$ & \\
& Negative & 29 & $22.5 \%$ & \\
\hline
\end{tabular}

${ }^{1} P$ value from chi-square goodness of fit test.

*Higher observed number respect to expected (all categories equal).

factors positively associated with high BCS. BCS was also positively associated with the cleanliness of the bedding (81.0\% of the camels living in pens with dirty bedding had low BCS; $P=0.028$ ). Finally, high BCS tended to be negatively associated with the presence of a disease $(P=0.059)$ and limited feeding space $(73.3 \%$ of camels with a low BCS had a feeding space $<0.40 \mathrm{~m}^{2} ; P=0.089$; Tables $\left.1 \mathrm{SM}\right)$.

Limited space allowance $(P<0.001)$ and shaded space $(P=0.016)$ were instead factors strongly associated with the highest (worst) thirst index. A high thirst index score was also positively associated with limited feeding $(P=0.032)$ and water space $(P=0.006)$. Conversely, a high thirst index was negatively associated with water temperature $(P=0.009)$. Finally, the odds of having a high thirst index score tended to increase as the number of camels per pen increased $(P=0.060)$ and in pens managed by well-experienced caretakers $(P=0.099$; Tables $2 \mathrm{SM})$.

\section{Good housing (Fig. 2a and b)}

Resting behaviors were positively associated with water temperature $(P=0.007)$ and rationed distribution of water $(P=0.041)$ as well as with the longest experience of the caretakers $(P=0.007)$. Resting behaviors also tended to be positively associated with dirty water $(P=0.065)$, the presence of hobbles on the camel $(P=0.095)$, and clean bedding $(P=0.054)$; namely, the odds of showing resting behavior was 3 times higher among camels living in pens with clean bedding (Table 3SM).

The likelihood of having a greater proportion of animals showing restricted movements was higher in pens holding camels reared for milk, breeding, or race $(5.3 \pm 2.1 \% ; P=0.001)$ and dual-purpose $(2.6 \pm 1.4 \%$; $P=0.038)$ than in pens with camels reared for meat production $(0.5 \pm 0.3 \%)$. The proportion of camels unable to move freely were also positively associated with ad libitum distribution of water $(P=0.016)$ while only a trend was found for water point positioned in the sun $(P=0.067$; Table 4SM).

\section{Good health (Figs. 3a, b, and 4)}

Regarding factors associated with the presence of disease, camels having a disease were 2.7 times more likely to live in pens with dirty bedding $(P=0.048)$. The percentage of healthy camels was only $41.5 \%$ in pens with dirty beddings while it rose to $65.3 \%$ in pens with clean or partially clean bedding. The other factors associated with increased odds of being sick were the rationed distribution 
Fig. 1 Forest plots displaying the associations between high body condition score (panel a) and thirst index (panel b) assessed at animal level and age, management-, or resourcebased measures in the pens of a camel market
Fig. 2 Forest plots displaying the associations between resting behaviors assessed at animal level (panel a) or restricted movement assessed at herd level (panel b) and management- or resource-based measures in the pens of a camel market

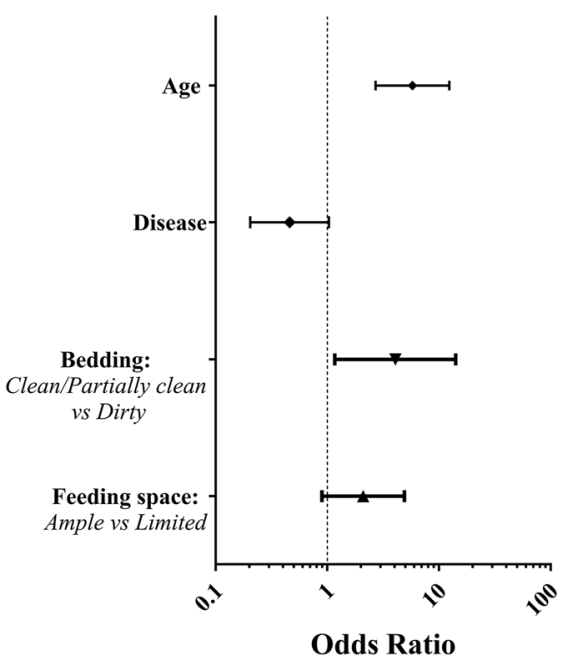

a

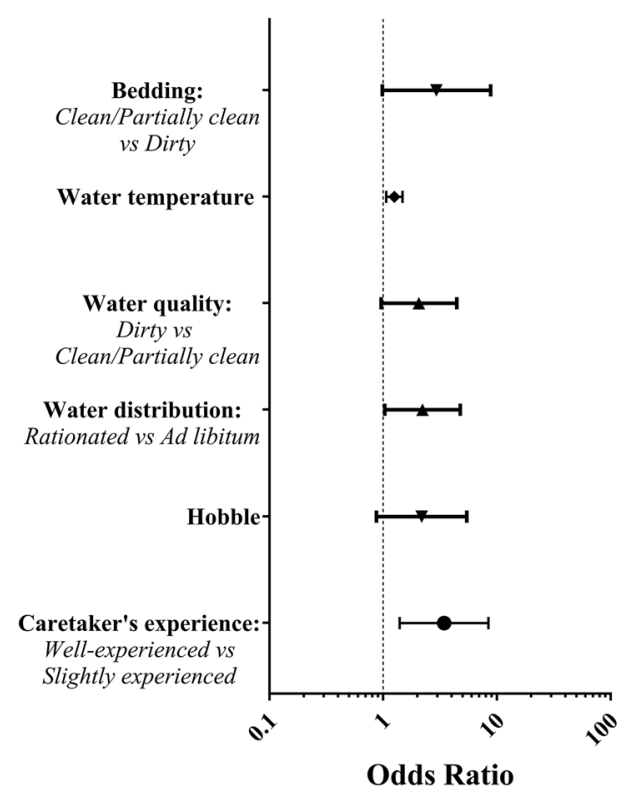

a

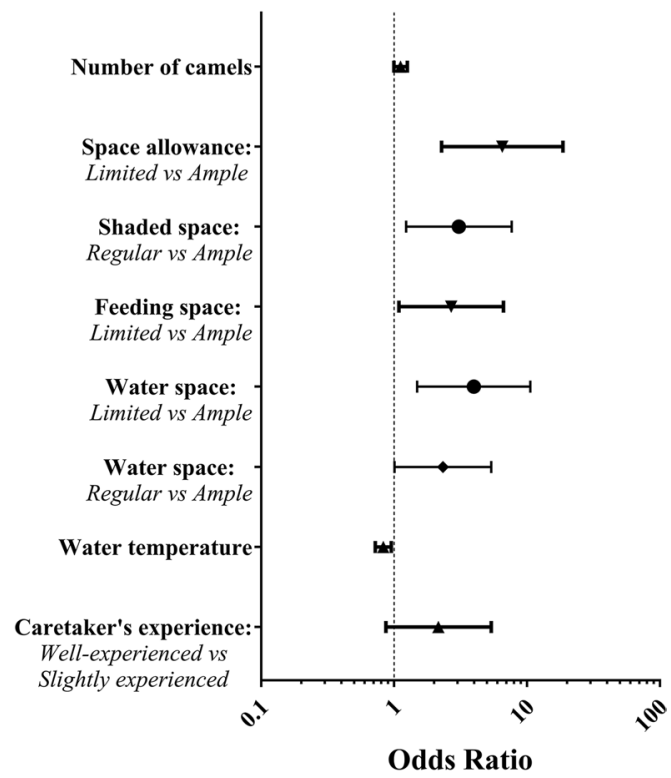

b

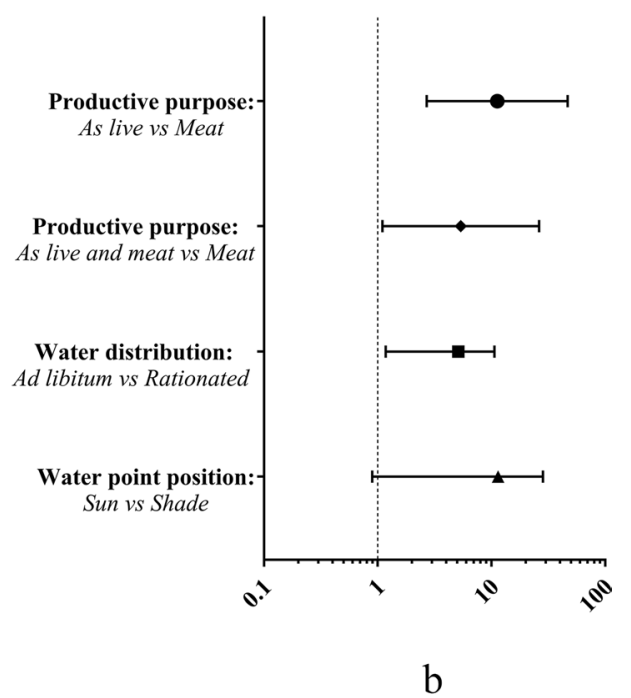

of water $(P=0.050)$ and the slight experience of the caretaker $(P=0.025)$. Indeed, nearly two-thirds of sick camels $(64.7 \%)$ did not receive water ad libitum while more than half of sick camels (53.6\%) were handled by slightly experienced caretakers. Finally, "disease" was negatively associated with the presence of hobbles $(P=0.032$; Table 5SM).

Camels with injuries were 2.7 times more likely to have hobbles than those without injuries (prevalence of injury: $37.0 \%$ and $18.1 \%$ among camels with and without hobbles, respectively; $P=0.038$ ). The other factors associated with increased odds of having injuries were the age of camels $(P=0.007)$ and moderate caretaker's experience $(P=0.042$; Table 6SM).

Protective factors for pain induced by management procedures were ample space allowance $(P=0.013)$, ample shaded $(P=0.003)$, feeding $(P<0.05)$, and water spaces $(P<0.05)$. For example, the estimated proportions of animals showing signs of pain induced by management procedures were $21.3 \pm 3.9 \%$ and $11.8 \pm 1.8 \%$ in pen with 
Fig. 3 Forest plots displaying the associations between disease (panel a) or injuries (panel b) assessed in camels at animal level and age, management- or resourced-based measures recorded in the pens of a camel market

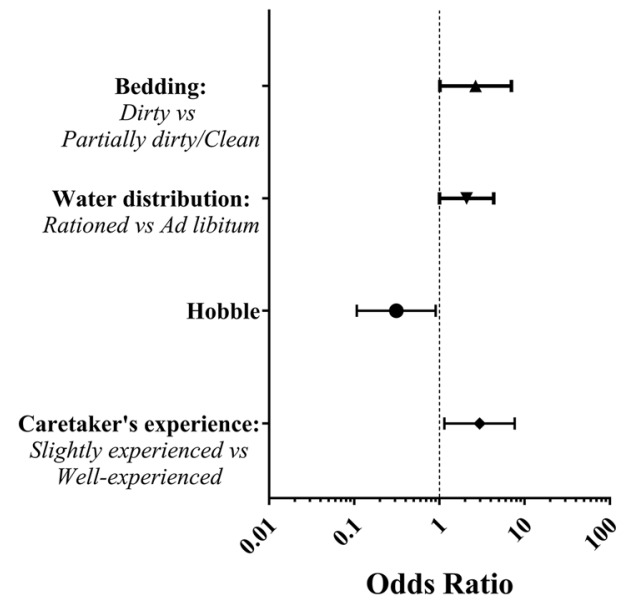

a

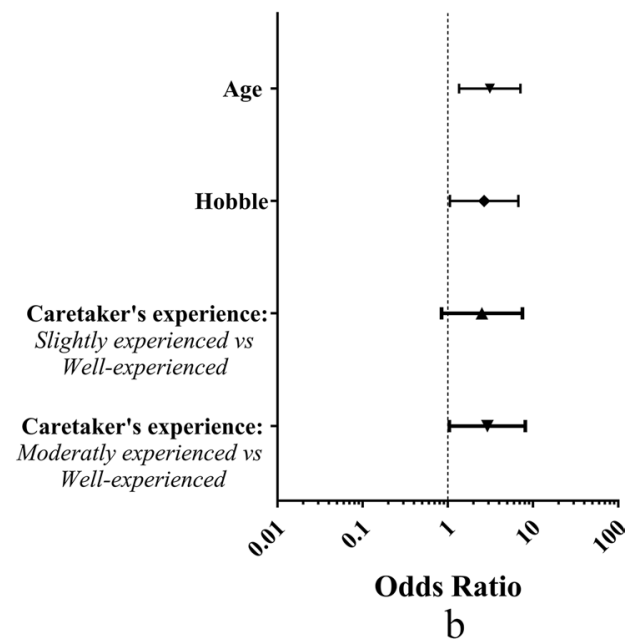

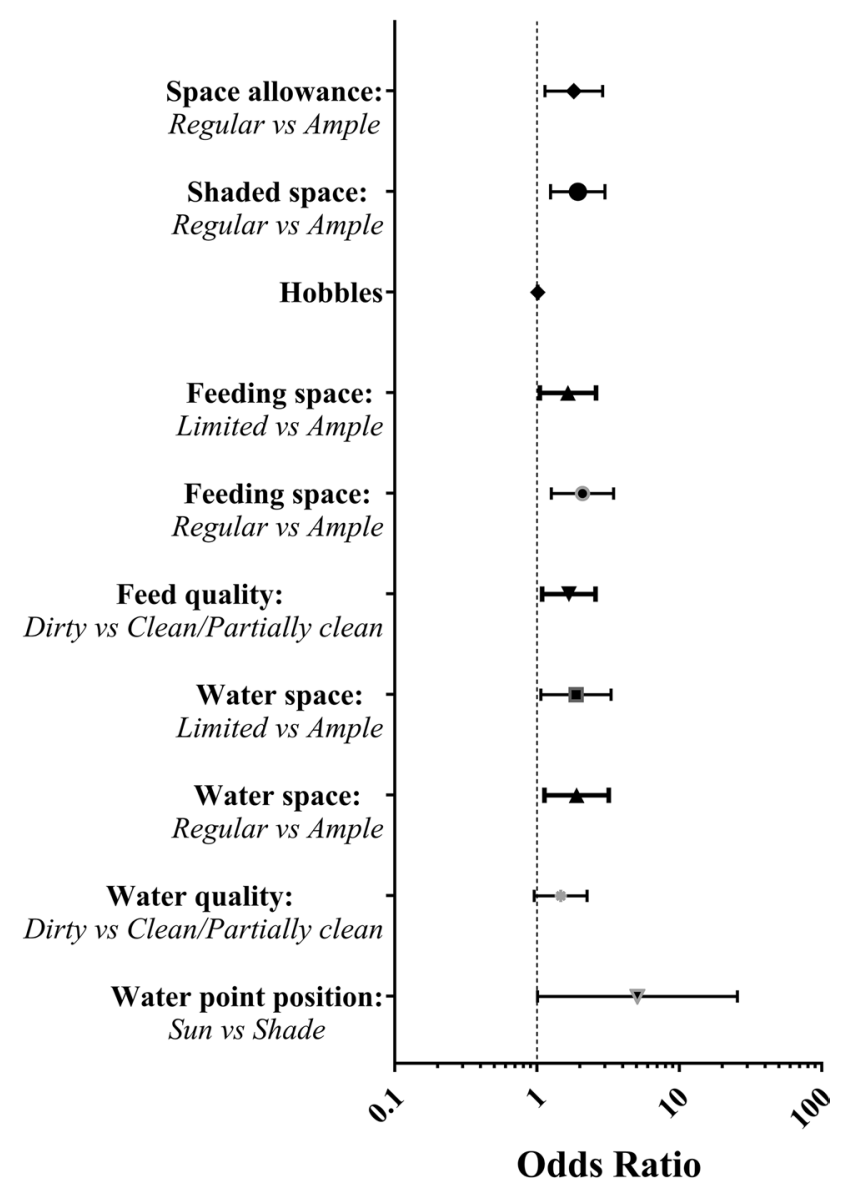

Fig. 4 Forest plot displaying the associations between indicators of pain induced by management procedures and management- or resource-based measures in the pens of a dromedary camel market

regular and ample space allowance, respectively, while they were $23.1 \pm 3.6 \%$ and $12.0 \pm 1.9 \%$ in pens with regular and ample shaded space, respectively. The presence of pain induced by management procedures was also positively associated with dirty feed $(P=0.020)$ and the placement under the sun of water points $(P=0.049)$. Moreover, animals showing pain induced by management procedures increased as the proportion of hobbled animals increases $(P<0.001)$. Finally, the presence of paininduced tended to be positively associated with dirty water $(P=0.081$; Table 7SM $)$.

\section{Appropriate behavior (Fig. 5a and b)}

Regarding factors associated with the approaching test, the likelihood of having negative responses reduced as camel age increased and this effect was significant both at the univariable and multivariable models (for all, OR $<1.00 ; P<0.05)$. Moreover, the negative response to the approaching test was positively associated with limited shaded space $(P=0.019)$ when compared with ample space. Indeed, about $35.0 \%$ of camels with limited shaded space showed negative responses while only $33.0 \%$ showed a positive response. Conversely, a positive response was recorded in over $60 \%$ of the camels having ample shaded space. Finally, responses to the approaching test tended to be affected by limited feeding space $(P=0.062)$ and rationed distribution of water $(P=0.095)$; namely, $82.8 \%$ of camels showing a negative response were not fed ad libitum (Table 8SM).

The presence of aggressive animals was positively associated with the number of camels in the pen $(P=0.002)$ and with limited space allowance $(P=0.029)$. In $54.2 \%$ of the pens where there were aggressive animals, indeed, the space allowance was $\leq 19.0 \mathrm{~m}^{2} /$ animal. Similarly, the presence of aggressive animals was positively associated with limited feeding $(P=0.021)$ and water space $(P=0.023)$. About half of the pens keeping aggressive animals had a feeding space $\leq 0.40 \mathrm{~m}^{2} /$ camel $(50.0 \%)$ and a water space $\leq 0.06 \mathrm{~m}^{2} /$ camel (45.8\%; Table 9SM). 
Fig. 5 Forest plots displaying the associations between negative responses to the approaching test of the camels assessed at animal level (panel a) or presence of aggressive camels in the pen (panel b) and managementor resource-based measures in the pens of a camel market
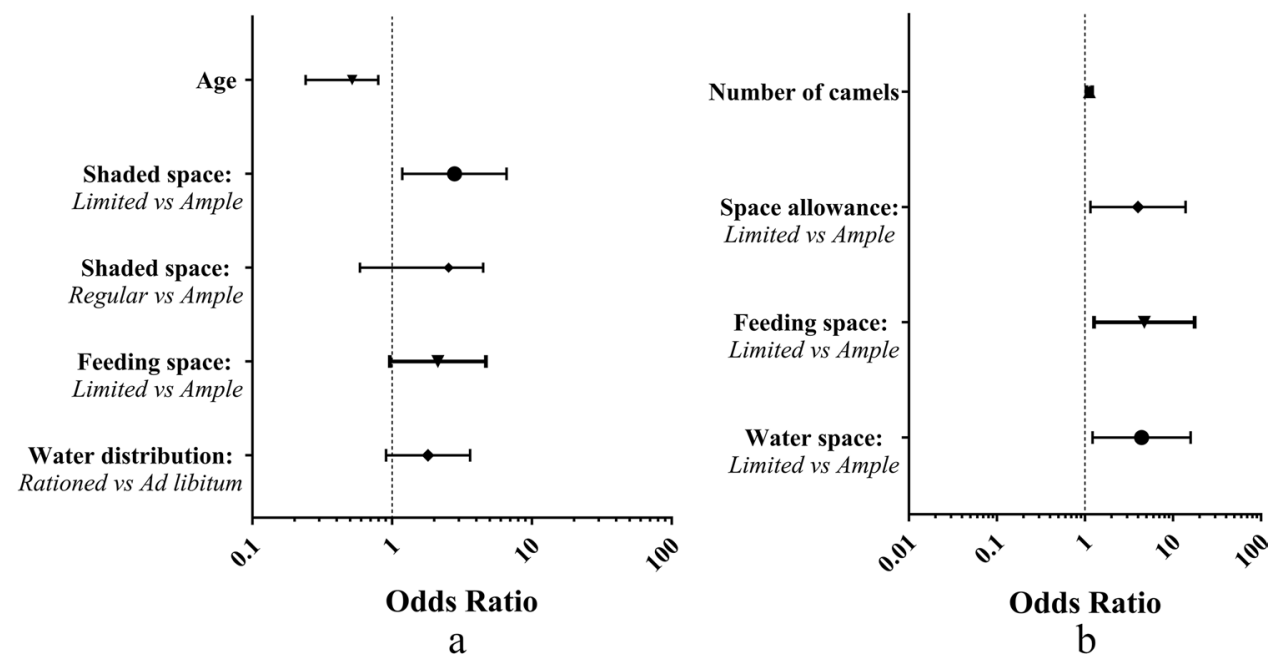

Figure 6 summarizes resource and management-related factors significantly associated with welfare outcomes of camels kept at the permanent market.

\section{Discussion}

This study proposed new ABMs for camels and evaluated their associations with resource and management-related factors in dromedary camels kept in a permanent market.
Our methodological approach, although did not presume to define direct cause-effect relationships, could verify the validity of some ABMs as welfare indicators as well as identify relevant factors related to housing and management that were likely linked to camel welfare. Thus, our findings may be useful to recognize welfare issues and propose guidelines to safeguard welfare in camels kept in intensive systems.

The space allowance and the number of camels per pen stood out among the management factors affecting

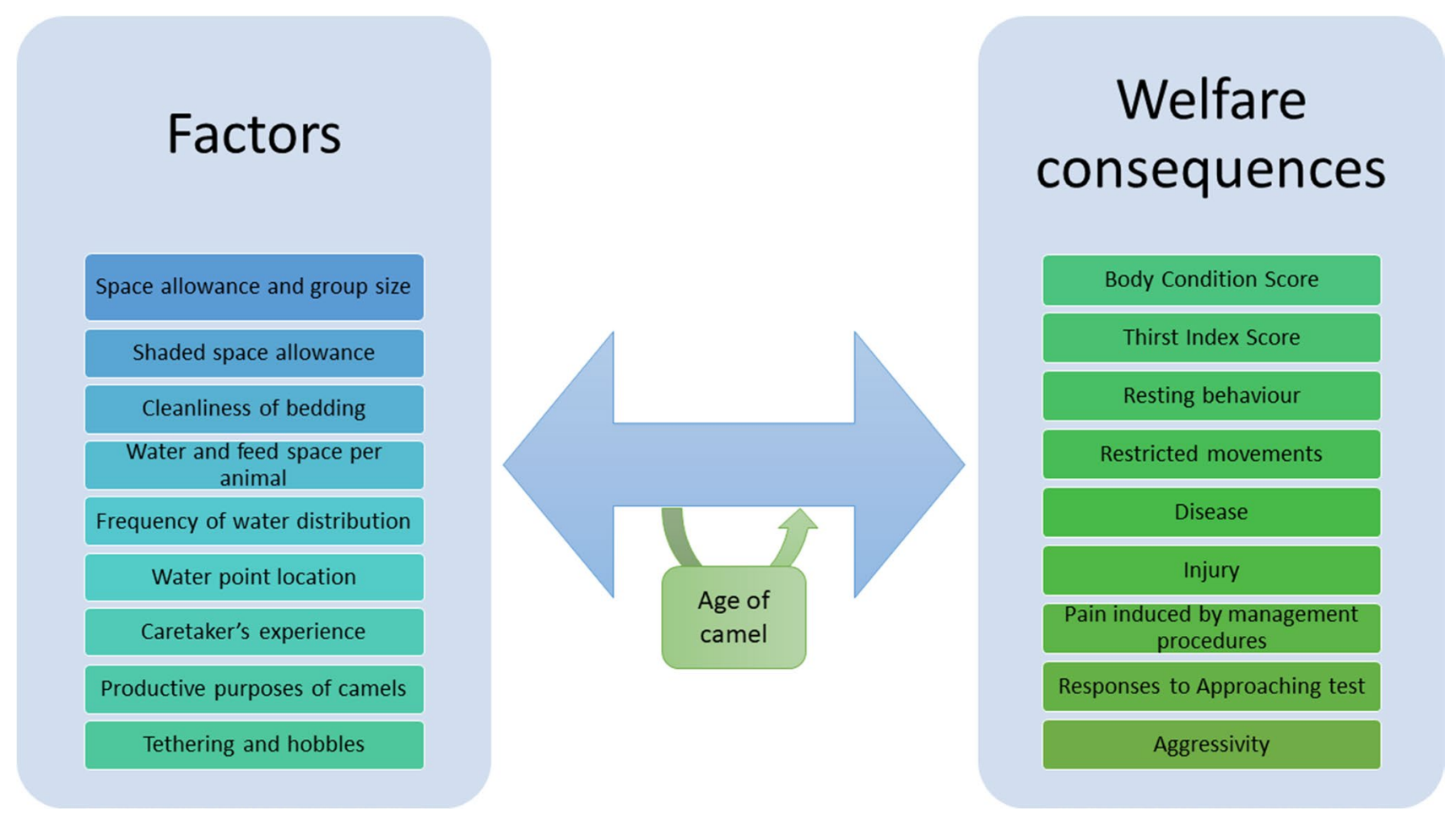

Fig. 6 An overview of management- and resource-based measures, treated as factors that may have the potential to influence dromedary camel welfare, associated with the animal-based measures, treated as indicators of welfare consequences 
camel welfare, being associated with many of the proposed ABMs. In pens where space allowance was less than $19 \mathrm{~m}^{2}$ per animal, indeed, camels had the worse thirst index as well as the highest likelihood of showing pain induced by management procedures and aggressive behaviors. These associations may be linked to both logistic and social factors. First, water and feed supply in overcrowded pens are more demanding from a management point of view, and besides, require facility adjustments. The probability, therefore, that water and feed needs are not fulfilled in overcrowded pens could be greater. Second, camels in overcrowded pens have limited opportunity to walk freely and frequently experience unstable social relationships. In this context, natural behaviors are inhibited (Wang et al., 2016) and dynamic dominance hierarchies characterized by high levels of agonistic behaviors could increase (Fu et al., 2016; Eriksson et al., 2021). Under natural conditions, indeed, camels move over very large areas and are great walkers, practising "ambulatory grazing" (Faye, 2020). Camels, moreover, are herd animals but groups are, in general, stable and not large. They indeed comprised around 9 animals including one adult bull, adult females, sub-adults, and young (Wilson, 1990; Schulte and Klingel, 1991). The risk of not meeting these ethological needs in intensive systems and livestock markets increases dramatically and, therefore, preventive measures are necessary. Our results indicated that a minimum space allowance of $19 \mathrm{~m}^{2}$ per camel could reduce the hazard of the negative welfare consequences. Caretakers, furthermore, should avoid the formation of too numerous herds, paying attention to their composition in terms of age, sex, and relationships between members in order to limit competitive hierarchies and negative social interactions. The effect of space allowance on camels' welfare has been recently also evaluated by El Shoukary et al. (2020) and Zappaterra et al. (2021). Those authors showed that overcrowding had negative effects not only on behavior, increasing aggressions, and stereotypies, but also on body conditions and reproductive performance of camels. Overcrowding is a recognized welfare concern for all the animals reared under intensive conditions (Nordquist et al., 2017). The adverse effect of limited space allowance on drinking and feeding behavior, abnormal behaviors, and body lesions have been indeed shown in most livestock species (SalakJohnson et al., 2012; Fu et al., 2016; Raspa et al., 2020; Eriksson et al., 2021).

Shaded space allowance was also associated with several proposed ABMs. Its link with the welfare criteria of absence of prolonged thirst, as evaluated by thirst index, is quite intuitive. However, our findings also showed that an ample shaded area was also associated with a reduction in the use of management procedures inducing pain (e.g., hobbles and nosering) and an improvement in the human-animal relationship. These findings may suggest that the presence of shade increased not only the thermal but also the social comfort of camels. It has recently been shown that camels have a preference for shade and express more behaviors indicating a quiet and positive state, such as recumbency and ruminating, in the shaded than sunny areas (Zappaterra et al., 2021). The opportunity to have positive experiences and express natural behaviors could have influenced their intra- and interspecies relationships resulting in an improvement of welfare outcomes. Moreover, if the shaded space is adequate for rearing density, camels could reduce competitive behavior and peacefully share this resource. Our results seem also to support Mellor's concept, which emphasizes the role of positive experiences in the animal welfare assessment (Mellor, 2016). According to Mellor, welfare cannot be only defined on the basis of the animals' responses to living in poor environments. It is also necessary to evaluate their opportunities to engage in behaviors they find rewarding, including thermal comfort, positive social interactions, resting, and feeding behaviors (Mellor, 2016). Behavioral changes related to the presence of shaded areas have been shown both in intensive and extensive systems in several species, such as cattle, sheep, buffaloes, and horses, although previous studies mostly focused on the effect on productive traits (Schütz et al., 2010; Holcomb et al., 2015; Giro et al., 2019; De et al., 2020; Mishra, 2021). A roof was present in most of the pens at the market suggesting that caretakers are aware of the negative impact of heat stress on the productivity and well-being of the camels. In several cases, however, the space allowance in the shade was limited. Our findings suggested that a shaded area of at least $7 \mathrm{~m}^{2}$ per animal (our ample category) had beneficial effects on several aspects of the camel's welfare but further studies should define specific recommendations according to the environmental heat load.

Bedding may affect animal welfare (Welfare Quality, 2009; AWIN, 2015). In our study, it was indeed associated with ABMs of good feeding, good housing, and good health. It is interesting to note that camels having a disease were about 3 times more likely to live in pens with dirty bedding confirming that keeping bedding clean is a good practice that should be recommended to prevent diseases also in camels (Schwartz and Dioli, 1992). Dermatitis and diarrhea were among the most frequently observed health problems in the market. Frequent cleaning and disinfection of the environment could minimize the presence of microorganisms as well as the spread of infectious disease, thus resulting in an effective preventive measure for these health problems. The role of bedding management on animal welfare has already been emphasized in other species such as cattle and horses (Quigley et al., 2017; Siegers et al., 2018; Robles et al., 2020). However, the magnitude of welfare consequences for camels could be very affected by climatic conditions. Their feces may indeed dry out quickly in the arid climate where 
they are usually raised (Padalino and Menchetti, 2021). Further studies, with longitudinal designs and in differentiated settings, could better define the role of bedding quality for camel welfare and validate additional indicators, possibly animal-based, to evaluate farm hygienic conditions. For instance, a score rating the cleanliness of some anatomical regions of camels (e.g., udder, legs, and flanks) rather than the bedding could be a more useful and direct welfare indicator. This type of score has already been proposed as welfare indicators for cattle resulting associated with several productive and pathological parameters such as milk somatic cell counts, mastitis, and enteritis (Faye and Barnouin, 1985; Ellis et al., 2007; Wolf et al., 2016; Molina et al., 2019).

Measures related to feeding and water management were other factors linked to many ABMs. In some cases, the link between these management factors and the ABMs was expected and easy to explain. Ample feeding and water spaces were, for example, associated with good body conditions and the absence of thirst. It is indeed logical to expect an increase in feeding and drinking activity as available spaces increase, particularly for those subordinate camels that may have more difficulty accessing resources due to their low social rank (Turner et al., 2000; DeVries et al., 2004). Ample feeding and water spaces were also associated with reduced risk of pain induced by management procedures and improved camel behavior. This finding confirms that increasing space allowance at the feed and water places could reduce competition and aggressive interactions among camels. As widely demonstrated in other species (Turner et al., 2000; DeVries et al., 2004), indeed, competition for resources is a primary reason for aggressive interactions in animals reared in groups under intensive systems. Sometimes, however, the relationship between management factors and ABMs could be less easy to explain. A management factor could just be a mediator or a confounder for other exposure variables; otherwise, different factors may interact to produce different animal-based responses (EFSA, 2012c). We could also hypothesize that the behavior of camels indirectly benefited from ample feeding and water spaces being mediated by the general improvement in body conditions. It has been shown, for example, that donkeys with a high BCS are friendlier with humans (Farhat et al., 2020). This hypothesis also supports the multidimensional concept of welfare in which biological functions, as indicated by BCS and thirst index, and affective state, as indicated by the absence of pain or fear, are connected and superimposed (Fraser, 2003). A feeding space $>1.10 \mathrm{~m}^{2} / \mathrm{camel}$ and a water space $>0.160 \mathrm{~m}^{2} /$ camel may be suggested to obtain good welfare outcomes related to principles of good feeding and health as well as of appropriate behaviors. However, these thresholds were calculated based on our study population and require external validation (Menchetti et al., 2021).

Ad libitum distribution of water and the placement of the water points in the shade are good husbandry practices
(Pritchard et al., 2005; Bergin and Nijman, 2019; Rodrigues et al., 2020). However, they were poorly followed in the market. The distribution of water was indeed rationed in about half of the pens and, for the most part, the water points were positioned in the sun. These factors were negatively associated with ABMs not only of good housing but also of good health. Water management is a well-known welfare issue for animals kept in several intensive and extensive contexts (Pritchard et al., 2005; Bergin and Nijman, 2019; Bekele et al., 2020; Rodrigues et al., 2020), but it may seem to be surprising for the camel due to its famous adaptability to resources scarcity. Thus, it is worthwhile noting that the odds of having a disease was 2 times higher in camels receiving rationed than ad libitum water suggesting that water supply is an aspect of camel welfare that cannot be overlooked (Menchetti et al., 2021; Padalino and Menchetti, 2021). It must also be taken into account that water needs in intensive systems are greater than in Bedouin ones (about 9 times higher to produce 11 of milk (Faye, 2013)). Our analyses also showed an inverse association between thirst and water temperature. In other words, non-thirsty camels were more likely to have hot water. This result was not expected and we can only speculate that the non-thirsty camels received water in overabundance and the excess water remaining in the trough increased its temperature over time. It, therefore, seems that the importance of having fresh water plays a secondary role compared to the benefits of ad libitum access.

Caretaker's experience and productive purpose of the camel resulted associated with the proposed ABMs. In particular, a long experience of caretakers seemed to be a protective factor for disease and injuries. This was expected since it has been already reported that the health of camels could benefit from staff training (Menchetti et al., 2021). Some health problems observed among camels, such as wounds, lameness, swollen joints, and mastitis, could benefit from more skilful and humane handling and welfare-friendly management of the animals. Regarding the productive purpose of camels, we curiously found that camels reared for milk or breeding were more likely to show signs of restricted movements than camels intended for slaughter. Perhaps the use of hobbles or ropes was more frequent in camel reared for milk or breeding as they needed to be more frequently moved and handled notably at milking time. The positive association between the presence of hobbles and ABMs such as pain or injuries is also worth mentioning to highlight the role of the limited freedom of movement and practices used for restraint on camel welfare. Limited freedom of movement has been already indicated as a factor that may have negative consequences from a social and metabolic point of view while the use of restraint tools is a direct cause of injuries, infections, and inflammations (Rayner et al., 2018; Farhat et al., 2020; Menchetti et al., 2021; Padalino and Menchetti, 2021). Therefore, when hobbles are considered 
unavoidable, it would be desirable to limit the use of inappropriate ropes, adopting also pain relief strategies, such as pads under the hobbles. Moreover, the camel's health status of camels wearing hobbles should be strictly monitored.

Finally, it is worth noting that the age of the camel was included in the applied statistical models as a covariate since it influenced several ABMs. For example, BCS increased as the camel's age increases, probably due to age-related changes in body composition (Kenyon et al., 2014; OucheneKhelifi and Ouchene, 2021). As expected, the likelihood of having injuries also increased with increasing camel's age while it appeared that aggressive behavioral responses to the human approach reduced over time. The accumulated familiarity with human contact and the taming could explain this last result (Waiblinger et al., 2006). The human-animal relationship could be indeed influenced by several factors but many of these are linked to the quality of past experiences. Habituation and early positive human contacts as well as the use of positive reinforcements are husbandry practices that could improve animal's perception of humans (Mota-Rojas et al., 2020) and should therefore be encouraged on camel farms. About a quarter of the camels at the market evaluated by the approaching test showed negative responses to human contact. Farm animals showing human-direct aggression and problematic to handle not only may lose their commercial value and be repeatedly sold but could also be manipulated with techniques that can exacerbate the undesirable behaviors causing injuries and stress. Aggressive animals could eventually be suppressed (Burattini et al., 2020). Conversely, a good human-animal relationship has positive effects not only on the welfare of the animal but also on its productivity and product quality (Waiblinger et al., 2006; Mota-Rojas et al., 2020). Despite this key role in animal welfare (Waiblinger et al., 2006; Welfare Quality, 2009; EFSA, 2012c), the human-animal relationship is complicated to assess and cannot be measured directly (Waiblinger et al., 2006). It probably deserves more valorization in the camel protocol and the validation of additional ABMs for its assessment (Padalino and Menchetti, 2021).

Our findings need to be interpreted with caution due to several limitations of the study. The main limitations are probably linked to the cross-sectional approach. For example, our study did not take into account the magnitude of welfare consequences (duration $\times$ intensity (EFSA, 2012c)) and could not provide definite information about cause-andeffect relationships. As suggested by EFSA, the duration of the welfare consequences might not even be considered in risk analysis whether the applied welfare measures reflect a momentary situation (EFSA, 2012a). A major criticism could be to treat the duration of management factors as constant. Camels could indeed be frequently moved or sold and this made the exposure to management factors very dynamic. Moreover, the quantitative indications concerning for example space allowance cannot be generalized, because these numbers were strictly related to our study population and require external validations as well as adjustments based on the type of scenario. It is also worth highlighting that among the categorical variables, the ABMs related to presence of a disease were limited by the type of assessments, which allows only to record the presence of major clinical signs. This could be overcame probably including more specific ABMs, able to identify the type of disease according to their original causes, and including longitudinal evaluations. As suggested by Faye et al. (1999), diseases can be classified into "immanent diseases," linked to the practices and conditions of the farms, and "transcendental diseases," coming from external causes (affecting all farming system independently of the practices and local environments, such as cases of Rift Valley Fever, MERS-Cov, anthrax, rabies, and brucellosis). This approach would clarify better the implications of the disease on camel welfare and how to prevent it. Finally, validation of the selected ABMs would require repeated measurements and the involvement of other researchers (EFSA, 2012c). Monitoring and surveillance systems inside the camels' pens/farms as well as a broad application of the welfare assessment protocol will help fill these gaps. Despite these limitations, the approach chosen in the selection of both animal-based and management-based measures complied with the feasibility features required for an on-farm welfare assessment (Main et al., 2007; EFSA, 2012a). These measures, moreover, seem "fit for purpose" (EFSA, 2012c) as identified several plausible welfare consequences as well as practicable corrective actions.

Overall, this study proposed new practical and feasible ABMs for dromedary camels kept under intensive management. They seemed to be appropriate indicators of welfare consequences in this species being able to identify factors related to housing and management practices that may impair or improve camel welfare. Our preliminary results need to be validated on a larger dataset, but they may be the first steps towards the development of welfare standards and guidelines for dromedary camels.

Supplementary Information The online version contains supplementary material available at https://doi.org/10.1007/s11250-021-02978-8.

Acknowledgements The authors would like to thank all camel caretakers and owners who participated in the study and the Ministry of Municipality and Environment of the State of Qatar for granting the approval.

Author contribution Conceptualization: Laura Menchetti, Barbara Padalino; methodology: Laura Menchetti, Barbara Padalino; formal analysis and investigation: Laura Menchetti; writing-original draft preparation: Laura Menchetti, Bernard Faye, Barbara Padalino; writing-review and editing: Bernard Faye, Barbara Padalino; funding acquisition Barbara Padalino; supervision: Barbara Padalino. 
Funding This study was funded by Animals' Angels.

Data availability All data are included in the article/SupplementaryMaterial; further inquiries can be directed to the corresponding author.

\section{Declarations}

Ethics approval The research project was approved by the Department for Agriculture Affairs \& Fisheries of the Ministry of Municipality and Environment of the State of Qatar (Approval code 2404/2020)

Conflict of interest The authors declare no competing interests.

\section{References}

Altman, D.G., 2014. Categorizing Continuous Variables Wiley StatsRef: Statistics Reference Online, 1-5

AWIN, 2015. Welfare assessment protocol for horses 1-80

Bauer, H. and Havenstein, J., 2017. The Welfare of Dromedary Camels during Road Transport in the Middle East (Animals' angels Publ: Francfort, Germany)

Bekele, T., Szonyi, B., Feleke, A. and Grace, D., 2020. Assessment of small ruminant welfare in Ethiopia-An abattoir-based study Journal of Applied Animal Welfare Science, 23, 356-365

Benaissa, M.H., Faye, B. and Kaidi, R., 2015. Reproductive abnormalities in female camel (Camelus dromedarius) in Algeria: Relationship with age, season, breed and body condition score Journal of Camel Practice and Research, 22, 67-73

Bengoumi, M. and Faye, B., 2002. Adaptation du dromadaire à la déshydratation Sécheresse (Montrouge), 13, 121-129

Bergin, D. and Nijman, V., 2019. An assessment of welfare conditions in wildlife markets across Morocco Journal of Applied Animal Welfare Science, 22, 279-288

Broom, D.M., 1996. Animal welfare defined in terms of coping with the environment Acta Agricultura Scandinavia, Supplement, $22-28$

Burattini, B., Fenner, K., Anzulewicz, A., Romness, N., McKenzie, J., Wilson, B. and McGreevy, P., 2020. Age-related changes in the behaviour of domestic horses as reported by owners Animals, $10,1-17$

Cook, N.B., 2017. Assessment of cattle welfare: Common animalbased measures Advances in Cattle Welfare, 27-53

De, K., Sharma, S., Kumawat, P.K., Kumar, D., Mohapatra, A. and Sahoo, A., 2020. Tree shade improves the comfort of sheep during extreme summer Journal of Veterinary Behavior, 40, 103-107

des Roches, A. de B., Veissier, I., Boivin, X., Gilot-Fromont, E. and Mounier, L., 2016. A prospective exploration of farm, farmer, and animal characteristics in human-animal relationships: An epidemiological survey Journal of Dairy Science, 99, 5573-5585

DeVries, T.J., Von Keyserlingk, M.A.G. and Weary, D.M., 2004. Effect of feeding space on the inter-cow distance, aggression, and feeding behavior of free-stall housed lactating dairy cows Journal of Dairy Science, 87, 1432-1438

Diverio, S., Boccini, B., Menchetti, L. and Bennett, P.C., 2016. The Italian perception of the ideal companion dog Journal of Veterinary Behavior: Clinical Applications and Research, 12, 27-35

EFSA, 2010. Animal welfare risk assessment guidelines on housing and management (EFSA Housing Risk) EFSA Supporting Publications, 7

EFSA, 2012a. Guidance on Risk Assessment for Animal Welfare EFSA Journal, 10, 2513
EFSA, 2012b. Scientific Opinion on the use of animal-based measures to assess welfare in pigs EFSA Journal, 10

EFSA, 2012c. Statement on the use of animal-based measures to assess the welfare of animals. Panel on Animal Health and Welfare (AHAW). EFSA Journal, 10, 2767

El Shoukary, R.D., Osman, A. and Mohammed, A., 2020. Effects of Stocking Density on Some Behavioral and Some Blood Biochemical Parameters in Camel during the Rut Period Egyptian Journal of Veterinary Sciences, 51, 253-262

Ellis, K.A., Innocent, G.T., Mihm, M., Cripps, P., McLean, W.G. and Howard, C.V., 2007. Dairy cow cleanliness and milk quality on organic and conventional farms in the UK Journal of Dairy Research, 74, 302-310

Eriksson, H.K., von Keyserlingk, M.A.G. and Weary, D.M., 2021. The effects of social environment on standing behavior and the development of claw horn lesions Journal of Dairy Science, 104, 2195-2211

Farhat, S.F., McLean, A.K. and Mahmoud, H.F.F., 2020. Welfare assessment and identification of the associated risk factors compromising the welfare of working donkeys (Equus asinus) in egyptian brick kilns Animals, 10, 1-17

Faye, B., 2013. Camel Farming Sustainability: The Challenges of the Camel Farming System in the XXIth Century Journal of Sustainable Development, 6, 74-82

Faye, B., 2020. Interaction between camel farming and environment In:, O. AlHaj , B. Faye , and R. D. Agrawal (eds), Handbook of research on health and environmental benefits of camel products, (Hershey, USA)

Faye, B., 2014. The Camel Today: Assets and Potentials Anthropozoologica, 49, 167-176

Faye, B. and Barnouin, J., 1985. Objectivation de la propreté des vaches laitières et des stabulations. L'indice de propreté Bulletin Technique, Centre de Recherches Zootechniques et Vétérinaires de Theix, 59, 61-67

Faye, B., Bengoumi, M., Cleradin, A., Tabarani, A. and Chilliard, Y., 2001. Body condition score in dromedary camel: A tool for management of reproduction Emirates Journal of Food and Agriculture, $13,1-6$

Faye, B., Waltner-Toews, D. and McDermott, J., 1999. From "ecopathology" to "agroecosystem health" Preventive Veterinary Medicine, 39, 111-128

Fraser, D., 2003. Assessing animal welfare at the farm and group level: The interplay of science and values Animal Welfare, 12, 433-443

Fu, L., Li, H., Liang, T., Zhou, B., Chu, Q., Schinckel, A.P., Yang, X., Zhao, R., Li, P. and Huang, R., 2016. Stocking density affects welfare indicators of growing pigs of different group sizes after regrouping Applied Animal Behaviour Science, 174, 42-50

Giro, A., Pezzopane, J.R.M., Barioni Junior, W., Pedroso, A. de F., Lemes, A.P., Botta, D., Romanello, N., Barreto, A. do N. and Garcia, A.R., 2019. Behavior and body surface temperature of beef cattle in integrated crop-livestock systems with or without tree shading Science of the Total Environment, 684, 587-596

Holcomb, K., Tucker, C. and Stull, C., 2015. Shade use by small groups of domestic horses in a hot, sunny environment Journal of Animal Science, 93, 5455-5464

Kenyon, P.R., Maloney, S.K. and Blache, D., 2014. Review of sheep body condition score in relation to production characteristics New Zealand Journal of Agricultural Research, 57, 38-64

Main, D.C.J., Whay, H.R., Leeb, C. and Webster, A.J.F., 2007. Formal animal-based welfare assessment in UK certification schemes Animal Welfare, 16, 233-236

Mellor, D.J., 2016. Updating animal welfare thinking: Moving beyond the "five freedoms" towards "A lifeworth living" Animals, 6, 21

Menchetti, L., Monaco, D., Ziani, A. and Padalino, B., 2020. Camel welfare: the first survey on camel caretakers' perspective Journal of Camelid Science, Paper accepted 
Menchetti, L., Zappaterra, M., Costa, L.N. and Padalino, B., 2021. Application of a protocol to assess camel welfare: Scoring system of collected measures, aggregated assessment indices, and criteria to classify a pen Animals, 11, 1-24

Mishra, S.R., 2021. Thermoregulatory responses in riverine buffaloes against heat stress: An updated review Journal of Thermal Biology, 96, 102844

Molina, L., Agüera, E., Maroto-Molina, F. and Pérez-Marín, C.C., 2019. Assessment of on-farm welfare for dairy cattle in southern Spain and its effects on reproductive parameters Journal of Dairy Research, 86, 165-170

Mota-Rojas, D., Broom, D.M., Orihuela, A., Velarde, A., Napolitano, F. and Alonso-Spilsbury, M., 2020. Effects of human-animal relationship on animal productivity and welfare Journal of Animal Behaviour and Biometeorology, 8, 196-205

Munoz, C., Campbell, A., Hemsworth, P. and Doyle, R., 2018. Animalbased measures to assess the welfare of extensively managed ewes Animals, 8, 2

Nordquist, R.E., van der Staay, F.J., van Eerdenburg, F.J.C.M., Velkers, F.C., Fijn, L. and Arndt, S.S., 2017. Mutilating procedures, management practices, and housing conditions that may affect the welfare of farm animals: Implications for welfare research Animals, 7

OIE, 2008. Introduction In:, Terrestrial Animal Health Code, (Paris)

Ouchene-Khelifi, N.A. and Ouchene, N., 2021. Relationships between age, body measurements, and testicular measurements in Arabia bucks in Algeria Tropical Animal Health and Production, 53, 91

Padalino, B., Aubé, L., Fatnassi, M., Monaco, D., Khorchani, T., Hammadi, M. and Lacalandra, G.M., 2014. Could dromedary camels develop stereotypy? The first description of stereotypical behaviour in housed male dromedary camels and how it is affected by different management systems PLoS ONE, 9, e89093

Padalino, B. and Menchetti, L., 2021. The first tool for assessing welfare of camels Frontiers in Veterinary Science, 7, 631876

Pastrana, C.I., González, F.J.N., Ciani, E., Capote, C.J.B. and Bermejo, J.V.D., 2020. Effect of research impact on emerging camel husbandry, welfare and social-related awareness Animals, 10, 780

Pritchard, J.C., Lindberg, A.C., Main, D.C.J. and Whay, H.R., 2005. Assessment of the welfare of working horses, mules and donkeys, using health and behaviour parameters Preventive Veterinary Medicine, 69, 265-283

Quigley, J.D., Hill, T.M., Deikun, L.L. and Schlotterbeck, R.L., 2017. Effects of amount of colostrum replacer, amount of milk replacer, and housing cleanliness on health, growth, and intake of Holstein calves to 8 weeks of age Journal of Dairy Science, 100, 9177-9185

Raspa, F., Tarantola, M., Bergero, D., Bellino, C., Mastrazzo, C.M., Visconti, A., Valvassori, E., Vervuert, I. and Valle, E., 2020. Stocking density affects welfare indicators in horses reared for meat production Animals, 10, 1-23

Rayner, E.L., Airikkala-Otter, I., Susheelan, A., Mellanby, R.J., Meunier, N. V., Gibson, A. and Gamble, L., 2018. Prevalence of mutilations and other skin wounds in working donkeys in Tamil Nadu, India Veterinary Record, 183, 450

Robles, I., Kelton, D.F., Barkema, H.W., Keefe, G.P., Roy, J.P., von Keyserlingk, M.A.G. and DeVries, T.J., 2020. Bacterial concentrations in bedding and their association with dairy cow hygiene and milk quality Animal, 14, 1052-1066

Rodrigues, J.B., Sullivan, R.J.E., Judge, A., Norris, S.L. and Burden, F.A., 2020. Quantifying poor working equid welfare in Nepalese brick kilns using a welfare assessment tool Veterinary Record, 187,445

Salak-Johnson, J.L., DeDecker, A.E., Horsman, M.J. and Rodriguez-Zas, S.L., 2012. Space allowance for gestating sows in pens: Behavior and immunity Journal of Animal Science, 90, 3232-3244

Schulte, N. and Klingel, H., 1991. Herd Structure, Leadership, Dominance and Site Attachment of the Camel, Camelus Dromedarius Behaviour, 118, 103-114

Schütz, K.E., Rogers, A.R., Poulouin, Y.A., Cox, N.R. and Tucker, C.B., 2010. The amount of shade influences the behavior and physiology of dairy cattle Journal of Dairy Science, 93, 125-133

Schwartz, H.J. and Dioli, M., 1992. The one-humped camel in Eastern Africa, H. j. Schwartz and M. Dioli (ed), (verlag josef margraf Scientific Books: Weikersheim, Germany)

Siegers, E.W., Anthonisse, M., van Eerdenburg, F.J.C.M., van den Broek, J., Wouters, I.M. and Westermann, C.M., 2018. Effect of ionization, bedding, and feeding on air quality in a horse stable Journal of Veterinary Internal Medicine, 32, 1234-1240

Tremolada, C., Bielińska, H., Minero, M., Ferrante, V., Canali, E. and Barbieri, S., 2020. Animal-based measures for the on-farm welfare assessment of geese Animals, 10, 890

Turner, S.P., Sinclair, A.G. and Edwards, S.A., 2000. The interaction of liveweight and the degree of competition on drinking behaviour in growing pigs at different group sizes Applied Animal Behaviour Science, 67, 321-334

Waiblinger, S., Boivin, X., Pedersen, V., Tosi, M.V., Janczak, A.M., Visser, E.K. and Jones, R.B., 2006. Assessing the human-animal relationship in farmed species: A critical review Applied Animal Behaviour Science, 101, 185-242

Wang, F.X., Shao, D.F., Li, S.L., Wang, Y.J., Azarfar, A. and Cao, Z.J., 2016. Effects of stocking density on behavior, productivity, and comfort indices of lactating dairy cows Journal of dairy science, 99, 3709-3717

Welfare Quality, 2009. Welfare Quality Assessment protocol for cattle Welfare Quality® Assessment Protocol for Cattle, 1-142

Wilson, R.T., 1990. Natural and man-induced behaviour ofthe onehumped camel Journalof Arid Environments, 19, 325-340

Wolf, R., Barkema, H.W., De Buck, J. and Orsel, K., 2016. Dairy farms testing positive for Mycobacterium avium ssp. Paratuberculosis have poorer hygiene practices and are less cautious when purchasing cattle than test-negative herds Journal of Dairy Science, 99, 4526-4536

Zappaterra, M., Menchetti, L., Nanni Costa, L. and Padalino, B., 2021. Do camels (Camelus dromedarius) need shaded areas? A case study of the camel market in Doha Animals, 11, 480

Publisher's note Springer Nature remains neutral with regard to jurisdictional claims in published maps and institutional affiliations. 